\title{
Vocal Tremor and Vibrato in the Same Person: Acoustic and Electromyographic Differences
}

Christopher Dromey

Brigham Young University, dromey@byu.edu

Marshall E. Smith

University of Utah

Follow this and additional works at: https://scholarsarchive.byu.edu/facpub

Part of the Communication Sciences and Disorders Commons

\section{Original Publication Citation}

Dromey, C. \& Smith, M.E. (2008). Vocal tremor and vibrato in the same person: acoustic and electromyographic differences. Journal of Voice, 22, 541-545.

\section{BYU ScholarsArchive Citation}

Dromey, Christopher and Smith, Marshall E., "Vocal Tremor and Vibrato in the Same Person: Acoustic and Electromyographic Differences" (2008). Faculty Publications. 1778.

https://scholarsarchive.byu.edu/facpub/1778

This Peer-Reviewed Article is brought to you for free and open access by BYU ScholarsArchive. It has been accepted for inclusion in Faculty Publications by an authorized administrator of BYU ScholarsArchive. For more information, please contact ellen_amatangelo@byu.edu. 
Vocal tremor and vibrato in the same person: acoustic and electromyographic differences

Christopher Dromey, Ph.D.

Brigham Young University

Provo, UT

Marshall E. Smith, M.D.

Division of Otolaryngology/Head and Neck Surgery

University of Utah School of Medicine

Salt Lake City, UT

Corresponding Author:

Christopher Dromey, Ph.D.

133 TLRB, Brigham Young University

Provo, UT 84602

Tel (801) 422-6461

Fax (801) 422-0197

dromey@byu.edu

Parts of this research were presented at the Annual Convention of the American SpeechLanguage-Hearing Association in San Diego, CA, November 2005. 
Abstract

Objectives: The goal of this study was to measure and describe differences between vocal vibrato and essential tremor of the voice in one individual who exhibited both types of modulation.

Study Design: Case study.

Methods: Recordings of spoken and sung vowels produced by the same individual at three effort levels were examined via analysis of acoustic and laryngeal electromyographic (LEMG) signals. Results: Modulation rate, periodicity and spectral measures of both audio and muscle activation signals revealed generally slower, more prominent and more regular patterns in sung than spoken conditions.

Conclusions: There was not always a clear correspondence between LEMG and acoustic measures, but both showed differences between the vibrato and tremor of this individual, suggesting differences in the neural bases of these modulations.

Keywords: vibrato, tremor, electromyography, acoustics 
Dromey \& Smith - Tremor and Vibrato Page 1

Introduction

Pathological vocal tremor and the artistic use of vibrato have long been topics of interest for researchers in the fields of speech science, medicine and singing. Several authors have considered the similarities and differences in these two vocal behaviors ${ }^{1-3}$. Because of perceptual parallels in the modulations that characterize these phenomena, similar acoustic analysis techniques have been applied to both. Tremor and vibrato introduce oscillations into an otherwise steady voice in the form of frequency (FM) and amplitude (AM) modulations. These typically vary in their rate from 4 to $7 \mathrm{~Hz}$. The extent of modulation ranges from barely perceptible to sufficiently severe to cause rhythmic arrests of voicing. Some authors have also examined the periodicity or regularity of vocal modulations ${ }^{1,4,5}$. These and other accounts have reported that vocal modulation in vibrato - especially FM - is basically sinusoidal, whereas pathological tremor is in many cases less smoothly periodic.

Fundamental to recent theories regarding the origin of these voice modulations is the notion of a mechanism for initiating and sustaining low frequency modulations in laryngeal muscle activity. Both peripheral and central mechanisms have been proposed. In the case of vibrato is has been hypothesized that a peripheral reflex loop with slowed conduction times and elevated gain may set up an agonist/antagonist resonance in the cricothyroid (CT) and thyroarytenoid (TA) muscles. This leads to rhythmic fluctuations in the tension of the laryngeal muscles ${ }^{6}$. The skilled singer can capitalize on these oscillations to produce "a pulsation of pitch, usually accompanied with synchronous pulsations of loudness and timbre, of such extent and rate as to give a pleasing flexibility, tenderness, and richness to the tones" 7 . Individuals with vocal tremor, on the other hand, may experience shaking as a consequence of a neurological condition such as Parkinson disease or multiple sclerosis. If their tremor is not secondary to another 
medical diagnosis, it is referred to as essential tremor, which can affect a number of structures, including the vocal tract in $15 \%$ of cases. A central neural oscillator is posited as the source of the tremor in all of these neurological conditions. The exact pathophysiology of tremor is not completely understood, in part because of the complexity of connections within the central nervous system. Different diseases may produce tremor by different mechanisms. The clinical manifestations of tremor vary in these conditions. However, metabolic studies in individuals with essential tremor have revealed abnormal cerebellar activation, and functional changes in the olivocerebellar circuit ${ }^{8,9}$, as well as rhythmic neuronal activation in the thalamus ${ }^{10}$.

A primary difference between the artistic and disordered conditions is that while vocal vibrato can be volitionally suppressed for a singer producing 'straight tone' vowels, the pathological condition is entirely involuntary. Indeed, while vibrato can even be modified in its rate $^{5}$, pathological vocal tremor is not responsive to attempts to change it by behavioral means. As a consequence, treatment is mostly limited to the use of laryngeal injection of botulinum toxin (BT) as a means to overcome the most disabling effects of this disorder ${ }^{11}$. However, since numerous anatomic structures can contribute to vocal modulation, BT injection into the vocal folds is not effective in reducing vocal tremor that is caused by respiratory or supralaryngeal vocal tract oscillations. Pharmacological agents have met with limited success ${ }^{12}$, and neurosurgical intervention, while effective in the regulation of disabling limb $\operatorname{tremor}^{13,14}$, is considered too invasive as a treatment for a voice disorder in the absence of limb involvement.

The present study is part of a larger investigation into the mechanisms of vocal tremor, involving LEMG and acoustic recordings. One of the individuals studied had a rapid tremor of the voice, which was present in sustained phonation as well as conversational speech. The reason for her inclusion in the present report is that she was also a singer accustomed to the use 
Dromey \& Smith - Tremor and Vibrato Page 3

of vibrato, and could produce 'sung' as well as 'spoken' vowels during data collection. These two types of phonatory behavior were characterized by modulations that differed perceptually, primarily in their rate. Key differences in the acoustic and muscle activation patterns are presented here to offer insights into the neural control of vocal modulation.

Method

The participant in this investigation was a 23 year old graduate student at the time of the study. She reported having experienced vocal tremor for about 5 years, and perceptual evaluation revealed a noticeable vocal unsteadiness during speech. When questioned regarding her vocal history, she reported having sung non-professionally since her teenage years. When she sang with vibrato, it was observed that her singing voice seemed to be modulated in a different way from her tremulous speaking voice.

As part of the larger IRB-approved study of vocal tremor, this speaker was recorded in a sound-treated booth with a head-mounted microphone (AKG-C420). Following local anesthesia of the skin overlying the cricothyroid space in the neck, hooked-wire electrodes were placed by the second author via percutaneous insertion into the left and right thyroarytenoid (TA) and cricothyroid (CT) muscles. Several non-speech maneuvers (swallow, cough, valsalva and highpitch phonation) were performed to verify the correct placement of the electrodes in the target muscles. Recordings were made of sustained vowel phonation in both 'spoken' and 'sung' modes. Three vowels were produced in a normal pitch and loudness condition, as well as at selfselected soft and loud effort levels. The targeting of specific loudness levels was not facilitated by any instrumental means, and pitch was not specified for these productions, because previous work has shown that the targeting of pitch and loudness can influence instrumental measures of 
Dromey \& Smith - Tremor and Vibrato Page 4

phonation ${ }^{15}$. The LEMG and microphone signals were pre-amplified and low-pass filtered at 5 $\mathrm{kHz}$ before being digitized at $10 \mathrm{kHz}$ with a Windaq (Dataq Instruments - Akron, $\mathrm{OH}$ ) analog to digital conversion system on a laboratory computer.

Binary files were exported from Windaq and imported into custom Matlab 7 routines ${ }^{16}$ for signal processing. Each analysis file consisted of 5 data channels (microphone, and LEMG for the right TA, left TA, right $\mathrm{CT}$ and left $\mathrm{CT}$ ). The three vowels in each condition were segmented visually from a waveform display and the acoustic channel was exported for each individual vowel for the computation of fundamental frequency $\left(\mathrm{F}_{0}\right)$ and amplitude modulation with Praat software ${ }^{17}$. The $\mathrm{F}_{0}$ and amplitude contours from Praat were imported back into Matlab for cross-correlation and modulation periodicity analysis along with the LEMG data. A 2 second window at the vowel temporal mid-point was used for the remaining analyses. The continuous amplitude of the EMG data channels was computed with a root-mean-square (RMS) operation, which resulted in a trace that was smoothed for plotting and further processing. Fast Fourier transforms (FFTs) were computed for each smoothed data channel to reveal the modulation rates of the signals. Pearson correlations were computed between the AM and FM acoustic modulations; they were also computed between either AM or FM and each EMG channel. A peak picking algorithm was used to identify each modulation cycle, and these data were used to calculate a coefficient of variation (COV - standard deviation divided by the mean, multiplied by 100) for each signal as an index of temporal irregularity in the modulations. Because the analysis of the 2 second segments was performed automatically by software algorithms, repeated processing yielded identical results, and thus testing to establish measurement errors was not conducted. 
Dromey \& Smith - Tremor and Vibrato Page 5

Results

Table 1 reports the frequency of modulation in the acoustic and LEMG signals as a function of loudness level. It also reports coefficients of variation in the same signals, and Pearson correlations between them. The values in the table represent the mean of three trials in each condition.

The impression of a difference in modulation between spoken and sung vowels was supported by these acoustic data. The rate of AM and FM in the spoken trials increased from soft to comfortable to loud far more than was the case for sung vowels (see Figure 1). The modulation rates of the EMG signals did not always match the acoustic findings. They were generally higher for the spoken than for the sung vowels. In the spoken vowels, the TA modulation rates were similar to the acoustic modulation in the loud condition, whereas the CT modulations were closer to the AM and FM rates for soft phonation. For singing, the CT modulation rates were generally more similar to the acoustic modulations than those from the TA muscles.

The COV measures were lower for singing than for speaking in the acoustic parameters of AM and FM (see Figure 2). The muscle activation modulations were more variable for the TA muscles for singing than for speaking in the loud condition. In the soft and comfortable conditions, they did not differ substantially between singing and speaking. The CT modulation COVs were only lower for singing than for speaking in the loud condition.

The AM and FM acoustic patterns were highly correlated for the sung vowels, but not for the spoken vowels (see Figure 3). The strength of this association changed little across the loudness conditions. The pattern of correlations between the acoustic and EMG signals was not straightforward. However, several observations may be made. The CT modulation was 
generally more strongly associated with the acoustic modulations in the sung than the spoken vowels. These were negative correlations, indicating that as the CT activation level was rising, the AM or FM signal was falling. The TA modulations were positively correlated with the acoustic modulations (more for FM than AM) for louder vowels, either spoken or sung.

Qualitative differences are visible in the acoustic and EMG modulation patterns for speaking and singing. Figure 4 shows that both F0 and left $\mathrm{CT}$ activity were modulated in a rhythmic fashion during loud singing. The FFTs show one dominant modulating frequency. However, Figure 5 shows the same measures, that is, F0 and LCT, for the loud spoken condition. The acoustic and EMG modulations are less regular, as well as less pronounced, and the muscle activity is modulated at multiple frequencies.

The F0 levels self-selected by the participant were higher for soft and loud than for comfortable effort trials, and were higher in each case for singing than for speaking.

\section{Discussion}

A number of studies have made reference to the similarities between vocal tremor and vibrato. While it is true that both are modulations of laryngeal output resulting from changes in muscle tension, the examination of both phenomena in the same individual has allowed valuable insights into some differences between them.

In the Western classical singing tradition, vibrato has generally been attributed to fluctuations in vocal fold tension, particularly as this results from modulation of CT activity ${ }^{18,19}$. Vocal tremor, on the other hand, has been noted to arise from a number of sources, including respiratory drive, laryngeal ab/adduction, pharyngeal wall instability, and tremor of the tongue, jaw or velum ${ }^{11,20-23}$. The diverse list of structures that can contribute to vocal tremor means that 
Dromey \& Smith - Tremor and Vibrato Page 7

the acoustic data do not always allow unambiguous inferences about the physiologic mechanisms in a given individual. However, the LEMG data allow at least some tentative insights into the differences between tremor and vibrato.

One observation that points to a difference in mechanisms for tremor and vibrato was that the modulation rate increased with loudness for spoken vowels, but not in the sung condition. Higher modulation rates for loud phonation have been reported previously in patients with vocal tremor ${ }^{4}$. It is possible that with higher effort phonation, the changes in muscle tension which result in tremor occur more quickly because the laryngeal muscles are more active. It could be speculated that muscles which are operating at a higher activity level respond more rapidly to the fluctuating levels of activation in the nerves that innervate them, and thus the modulation rate increases. However, the lack of modulation rate change in the sung conditions suggests that higher vocal intensity may be achieved in a different way from speaking, or perhaps more likely that the modulation in singing, being volitional, is regulated in such a way as to override the influence of overall increases in laryngeal muscle activity.

The higher modulation period COV values for spoken than for sung vowels reflect greater regularity in the vibrato than the tremor of this individual. The differences were most apparent in soft voice, and became less pronounced for louder phonation. The modulation in the acoustic and EMG signals was visibly stronger and more regular for sung than spoken conditions, as seen in Figures 4 and 5. The presence of a single dominant EMG peak in the vibrato stands in contrast to the multiple modulating frequencies in the spoken vowels. Previous reports have noted that there can be more than one modulating frequency in tremor ${ }^{1}$, and the data from the spoken vowels in the present study are consistent with this observation. While vocal tremor can be somewhat erratic in its patterns, listener judgments of vibrato quality have 
Dromey \& Smith - Tremor and Vibrato Page 8

favored voices with the greatest regularity in vibrato ${ }^{24}$. The participant in the present study was clearly able to switch between these two forms of vocal modulation.

The acoustic analysis revealed that AM and FM in the microphone recordings were correlated for sung but not for spoken vowels (see Figure 3). Thus for singing, instantaneous amplitude rose along with $\mathrm{F}_{0}$. Horii and colleagues ${ }^{19,25,26}$ presented a model of modulation in vibrato which they called the resonance-harmonics interaction. As singers modulate $\mathrm{F}_{0}$ by modulating the activity of the CT muscle, the harmonics rise and fall along with the $\mathrm{F}_{0}$. As these harmonics increase and decrease in frequency, they tend to coincide to a greater or a lesser degree with the resonant peaks in the vocal tract transfer function. Where harmonics and formants coincide, the acoustic output at the lips becomes stronger. Therefore, AM in singers can arise as an acoustic epiphenomenon as laryngeal harmonics interact with vocal tract formant peaks. This model would appear to support the present findings for singing, where AM and FM are clearly correlated. However, for speaking, the association is much weaker, suggesting that other mechanisms are responsible for these modulations.

Because muscle activity is responsible for changes in vocal fold tension and thus for acoustic modulation itself, one might expect a predictable association between LEMG activity and acoustic measures. However, the link between the two is not straightforward in the present data set. Horii's resonance-harmonics mode ${ }^{25}$ can explain the link between changes in $\mathrm{F}_{0}$ and changes in the radiated sound pressure level. However, $\mathrm{F}_{0}$ itself can change not only actively as a singer modulates the activity of the $\mathrm{CT}$, but also passively as a consequence of pulsations in subglottic pressure, as might occur in a respiratory-based vocal tremor. Because the present study focused on laryngeal muscle activity, we are not in a position to speculate about the 
possible role of other speech mechanism oscillations which might have influenced AM, FM, or both.

One limitation of the present study was that $\mathrm{F}_{0}$ levels were different for spoken and sung vowels in each loudness condition. Because the participant was free to self-select the pitch of her productions, no specific targets were requested or produced. Previous work has found that both vocal effort and pitch can influence vocal tremor ${ }^{4}$, and these influences may have interacted in this experiment. Had the tokens recorded here not been part of a much longer experimental protocol, it would have been possible to examine the influence of deliberate pitch and loudness changes in greater detail. Nevertheless, the data set does allow some valuable insights into differences between tremor and vibrato in the same individual.

Acknowledgments: This research was supported by K08-DC00132 from the National Institute of Deafness and Communication Disorders. 


\section{Reference List}

1. Buder EH, Strand EA. Quantitative and graphic acoustic analysis of phonatory modulations: The modulogram. J Speech Lang Hear Res 2003;46:475-90.

2. Ramig LA, Shipp T. Comparative measures of vocal tremor and vocal vibrato. J Voice 1987;1(2):162-7.

3. Winholtz WS, Ramig LO. Vocal tremor analysis with the vocal demodulator. $J$ Speech Hear Res 1992;35:562-73.

4. Dromey C, Warrick P, Irish J. The influence of pitch and loudness changes on the acoustics of vocal tremor. J Speech Lang Hear Res 2002;45:879-90.

5. Dromey C, Carter N, Hopkin A. Vibrato rate adjustment. J Voice 2003;17:168-78.

6. Titze IR, Story B, Smith M, Long R. A reflex resonance model of vocal vibrato. J Acoust Soc Am 2002 May;111(5 Pt 1):2272-82.

7. Seashore CE. The vibrato. Iowa City: University of Iowa; 1932.

8. Louis ED. Behavioral symptoms associated with essential tremor. Adv Neurol 2005;96:284-90.

9. Deuschl G, Raethjen J, Lindemann M, Krack P. The pathophysiology of tremor. Muscle Nerve 2001 June;24(6):716-35.

10. Hua SE, Lenz FA. Posture-related oscillations in human cerebellar thalamus in essential tremor are enabled by voluntary motor circuits. J Neurophysiol 2005 January;93(1):117-27.

11. Warrick P, Dromey C, Irish J, Durkin L, Pakiam A, Lang A. Botulinum toxin for essential tremor of the voice with multiple anatomical sites of tremor: A crossover design study of unilateral versus bilateral injection. Laryngoscope 2000;110:1366-74.

12. Busenbark K, Ramig L, Dromey C, Koller WC. Methazolamide for essential voice tremor. Neurology 1996 November;47(5):1331-2.

13. Goldman MS, Kelly PJ. Stereotactic thalamotomy for medically intractable essential tremor. Stereotact Funct Neurosurg 1992;58(1-4):22-5.

14. Goldman MS, Kelly PJ. Symptomatic and functional outcome of stereotactic ventralis lateralis thalamotomy for intention tremor. J Neurosurg 1992 August;77(2):223-9.

15. Hanson DG, Gerratt BR, Berke GS. Frequency, intensity, and target matching effects on photoglottographic measures of open quotient and speed quotient. J Speech Hear Res 1990;33(1):45-50.

16. Matlab [computer program]. Natick, MA: 2005. 
17. Praat [computer program]. 2005.

18. Imaizumi S, Saida H, Shimura Y, Hirose H. Harmonic analysis of the singing voice. Stockholm Music Acoustics Conference Proceedings; Stockholm: Royal Swedish Academy of Music; 1993 p. 197-201.

19. Horii Y. Frequency modulation characteristics of sustained /a/ sung in vocal vibrato. $J$ Speech Hear Res 1989 December;32(4):829-36.

20. Hachinski VC, Thomsen IV, Buch NH. The nature of primary vocal tremor. Can J Neurol Sci 1975;2:195-7.

21. Brown JR, Simonson J. Organic voice tremor. Neurology 1963;13:520-3.

22. Inbar GF, Eden G. Physiological evidence for central modulation of voice tremor. Biol Cybern 1983;47(1):1-12.

23. Ackermann H, Ziegler W. Cerebellar voice tremor: an acoustic analysis. $J$ Neurol Neurosurg Psychiatry 1991 January;54(1):74-6.

24. Robison CW, Bounous B, Bailey R. Vocal Beauty: A study proposing its acoustical definition and relevant causes in classical baritones and female belt singers. NATS Journal 1994;51:19-30.

25. Horii $\mathrm{Y}$, Hata $\mathrm{K}$. A note on phase relationships between frequency and amplitude modulations in vocal vibrato. Folia Phoniatr 1988;40:303-11.

26. Horii Y. Acoustic analysis of vocal vibrato: A theoretical interpretation of data. $J$ Voice 1989;3(1):36-43. 
Table 1. Acoustic and muscle activity modulation rates, irregularity indices, and inter-variable correlations for spoken and sung vowels as a function of vocal effort level.

\begin{tabular}{lrrrrrr} 
& Soft & Comf & Loud & Soft & Comf & Loud \\
\cline { 2 - 6 } AM Freq (Hz) & 3.6 & 4.0 & 7.0 & 4.8 & 5.0 & 5.3 \\
FM Freq (Hz) & 3.8 & 6.1 & 7.8 & 4.8 & 4.8 & 5.3 \\
RTA Freq (Hz) & 3.8 & 6.5 & 7.8 & 6.1 & 4.8 & 5.3 \\
LTA Freq (Hz) & 7.6 & 4.2 & 7.8 & 6.3 & 4.8 & 5.8 \\
RCT Freq (Hz) & 3.8 & 6.1 & 5.7 & 5.6 & 5.0 & 5.3 \\
LCT Freq (Hz) & 4.4 & 6.0 & 5.4 & 4.8 & 5.2 & 5.3 \\
& & & & & & \\
AM Period COV & 45.3 & 39.0 & 27.2 & 9.6 & 21.5 & 15.8 \\
FM Period COV & 40.2 & 36.1 & 16.5 & 12.8 & 18.7 & 10.0 \\
RTA Period COV & 35.0 & 28.5 & 14.0 & 30.7 & 35.4 & 28.2 \\
LTA Period COV & 36.4 & 34.9 & 14.8 & 33.7 & 32.5 & 29.8 \\
RCT Period COV & 36.0 & 25.3 & 30.7 & 28.7 & 25.5 & 24.2 \\
LCT Period COV & 30.2 & 27.8 & 27.5 & 30.7 & 27.2 & 18.0 \\
& & & & & & \\
AM FM Corr & 0.179 & 0.278 & 0.261 & 0.834 & 0.732 & 0.846 \\
AM RTA Corr & 0.081 & 0.062 & 0.285 & 0.063 & 0.104 & 0.449 \\
AM LTA Corr & 0.078 & -0.118 & 0.139 & -0.106 & -0.463 & -0.069 \\
AM RCT Corr & -0.160 & -0.211 & 0.024 & -0.296 & -0.456 & -0.554 \\
AM LCT Corr & -0.084 & -0.242 & 0.097 & -0.441 & -0.375 & -0.544 \\
FM RTA Corr & -0.060 & 0.169 & 0.435 & -0.006 & 0.072 & 0.493 \\
FM LTA Corr & 0.013 & 0.167 & 0.377 & -0.154 & -0.271 & 0.121 \\
FM RCT Corr & -0.127 & -0.389 & 0.134 & -0.430 & -0.542 & -0.548 \\
FM LCT Corr & -0.118 & -0.378 & 0.077 & -0.558 & -0.529 & -0.690 \\
F0 & & & & & & \\
& 356.4 & 278.4 & 390.6 & 517.1 & 479.0 & 533.8
\end{tabular}

Notes:

Comf = comfortable; $\mathrm{AM}=$ amplitude modulation; $\mathrm{FM}=$ frequency modulation; Freq = frequency; RTA = right thyroarytenoid; LTA = left thyroarytenoid; RCT = right cricothyroid; $\mathrm{LCT}=$ left cricothyroid; COV = coefficient of variation; Corr $=$ Pearson correlation; $\mathrm{F} 0=$ fundamental frequency 


\section{FIGURE CAPTIONS}

Figure 1. Amplitude (AM) and frequency (FM) modulation rates for vowels produced in spoken or sung conditions as a function of vocal effort level.

Figure 2. Modulation irregularity (measured as the coefficient of variation in the modulation period) for vowels produced in spoken or sung conditions as a function of vocal effort level.

Figure 3. Pearson correlations between the amplitude (AM) and frequency (FM) modulations in the acoustic signal for vowels produced in spoken or sung conditions as a function of vocal effort level.

Figure 4. Fundamental frequency and left cricothyroid activity modulation in the loud sung condition for a single vowel (upper panel) and FFT spectra of the acoustic (FM) and muscle (LCT) modulations in the lower panels. FFT y-axis in arbitrary units.

Figure 5. Fundamental frequency and left cricothyroid activity modulation in the loud spoken condition for a single vowel (upper panel) and FFT spectra of the acoustic (FM) and muscle (LCT) modulations in the lower panels. FFT y-axis in arbitrary units. 
Click here to download high resolution image

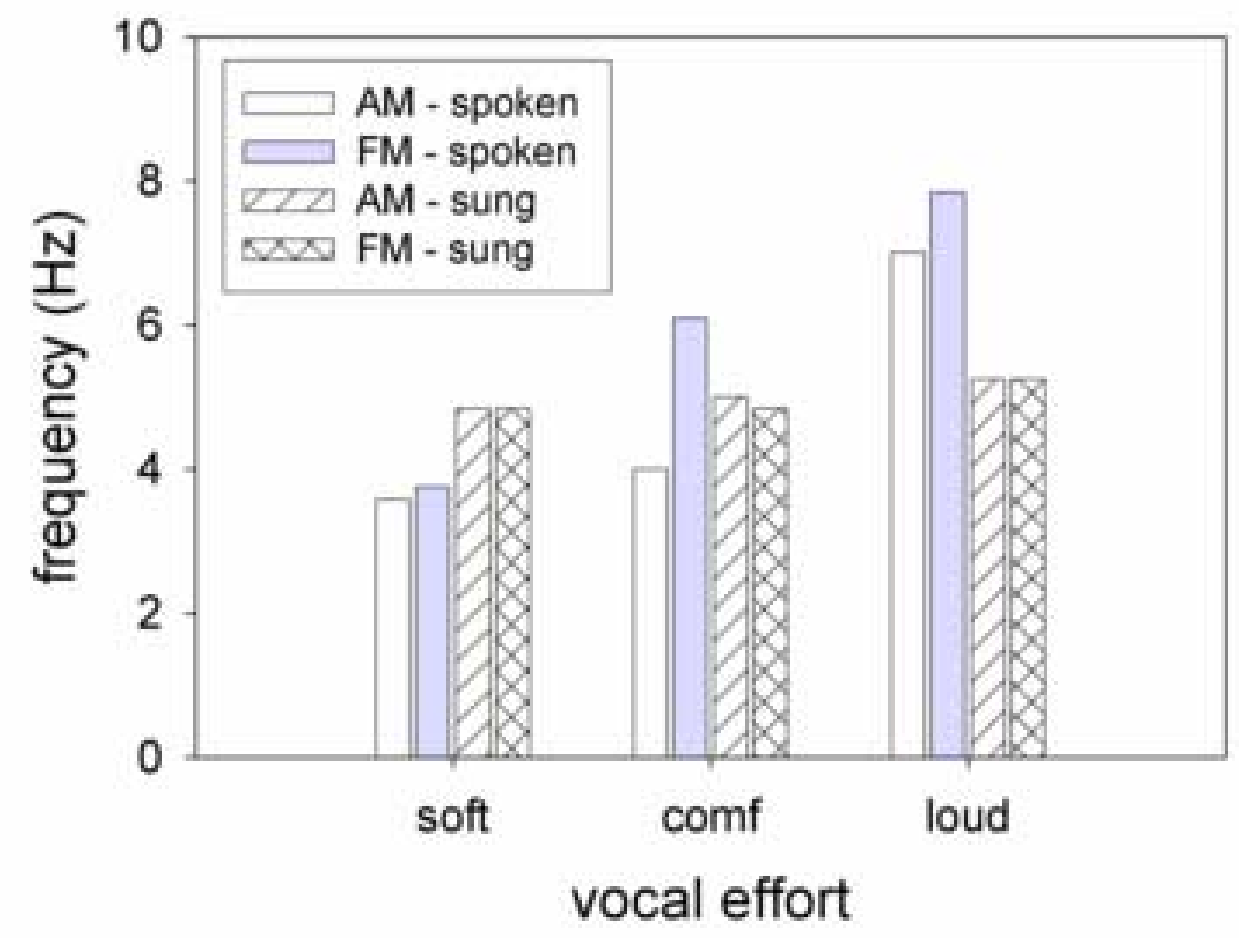


Click here to download high resolution image

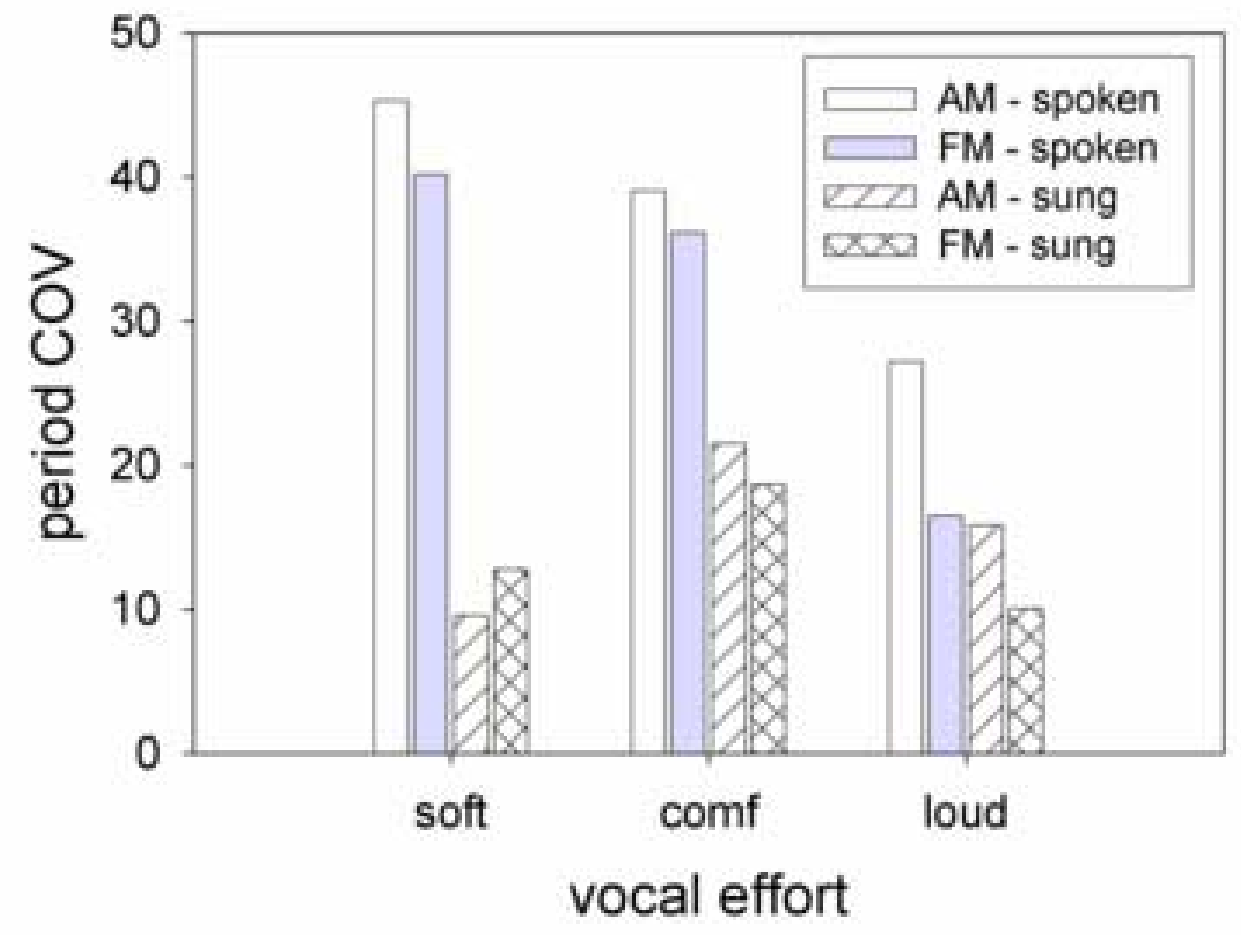


Click here to download high resolution image

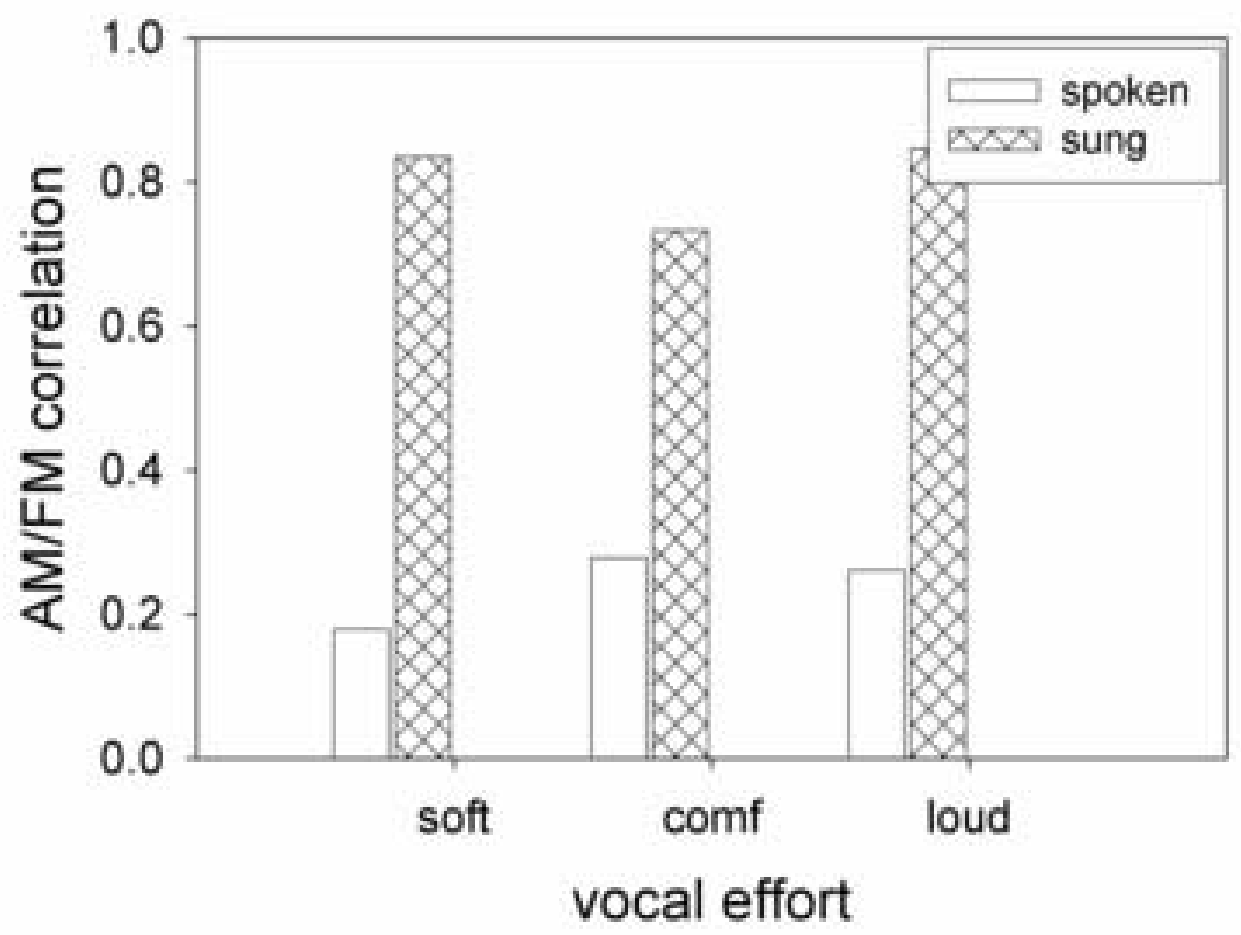




\section{Figure(s)}

Click here to download high resolution image

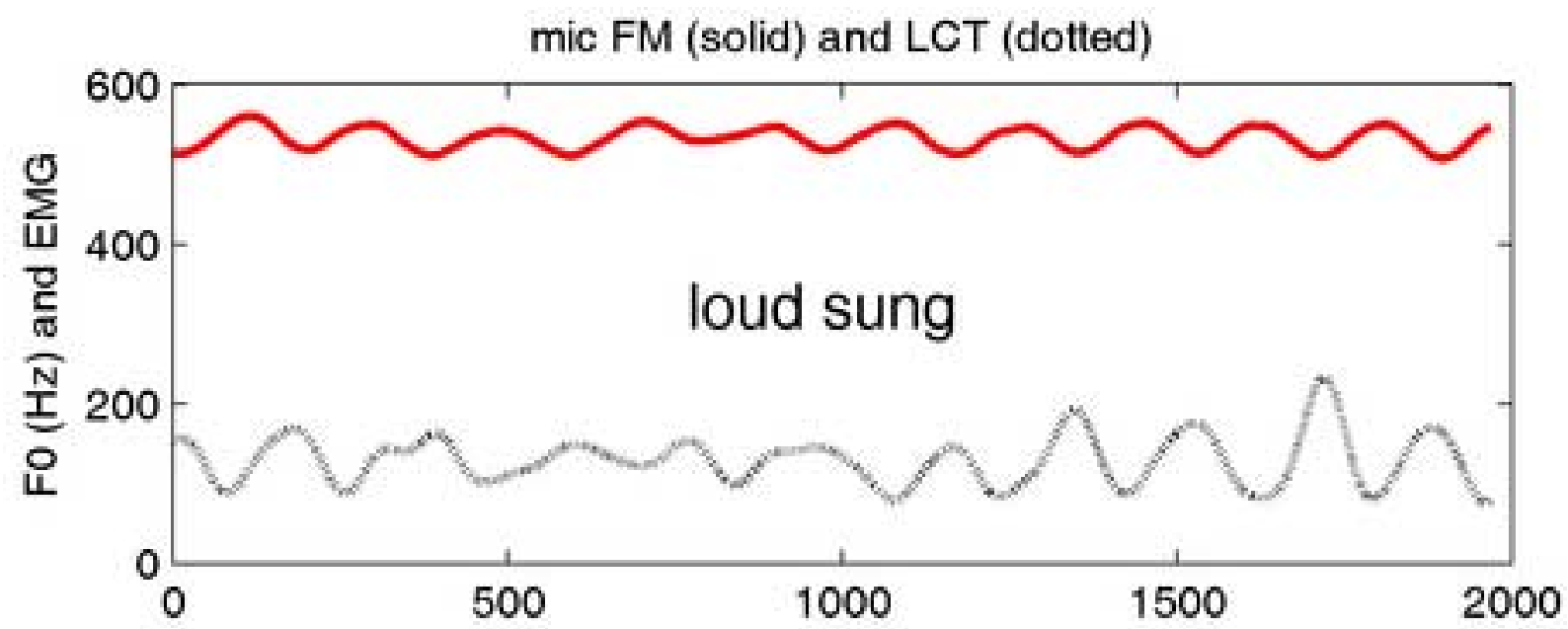

FM FFT
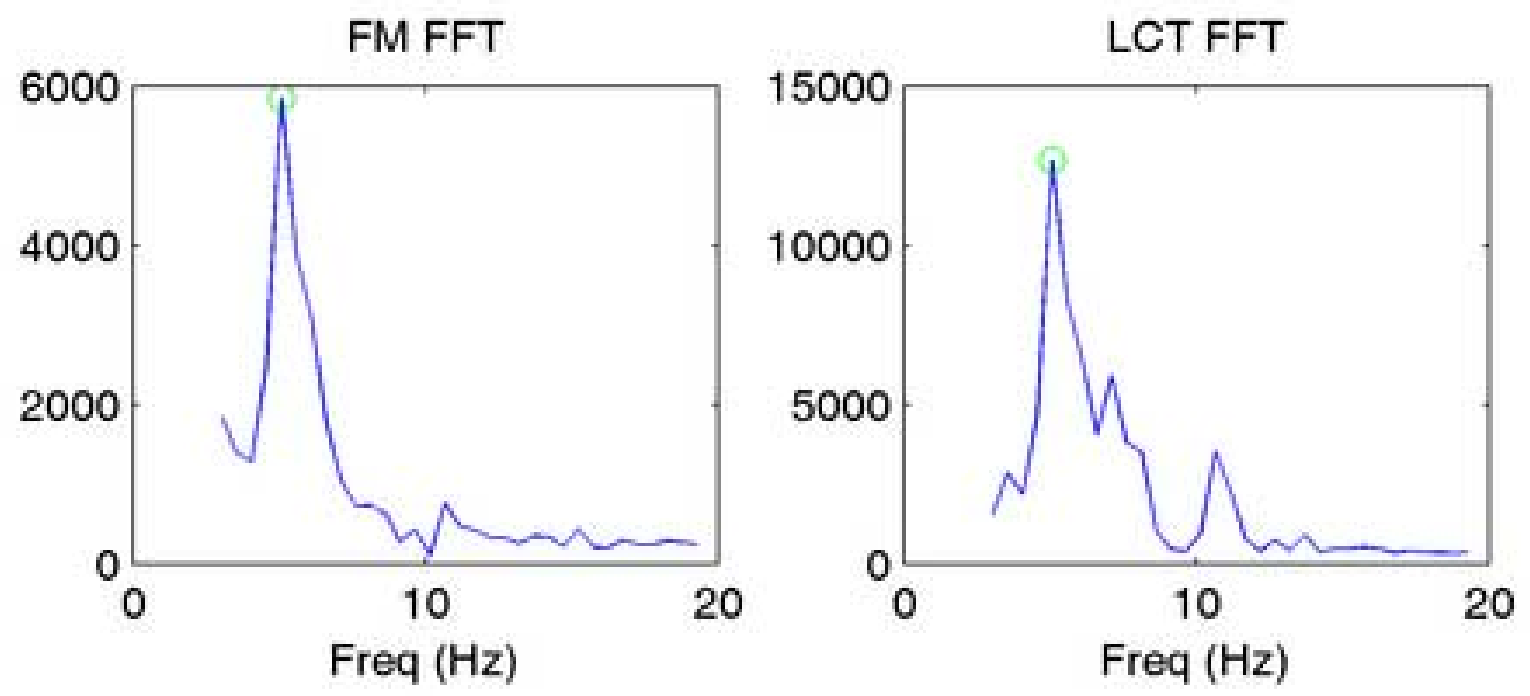

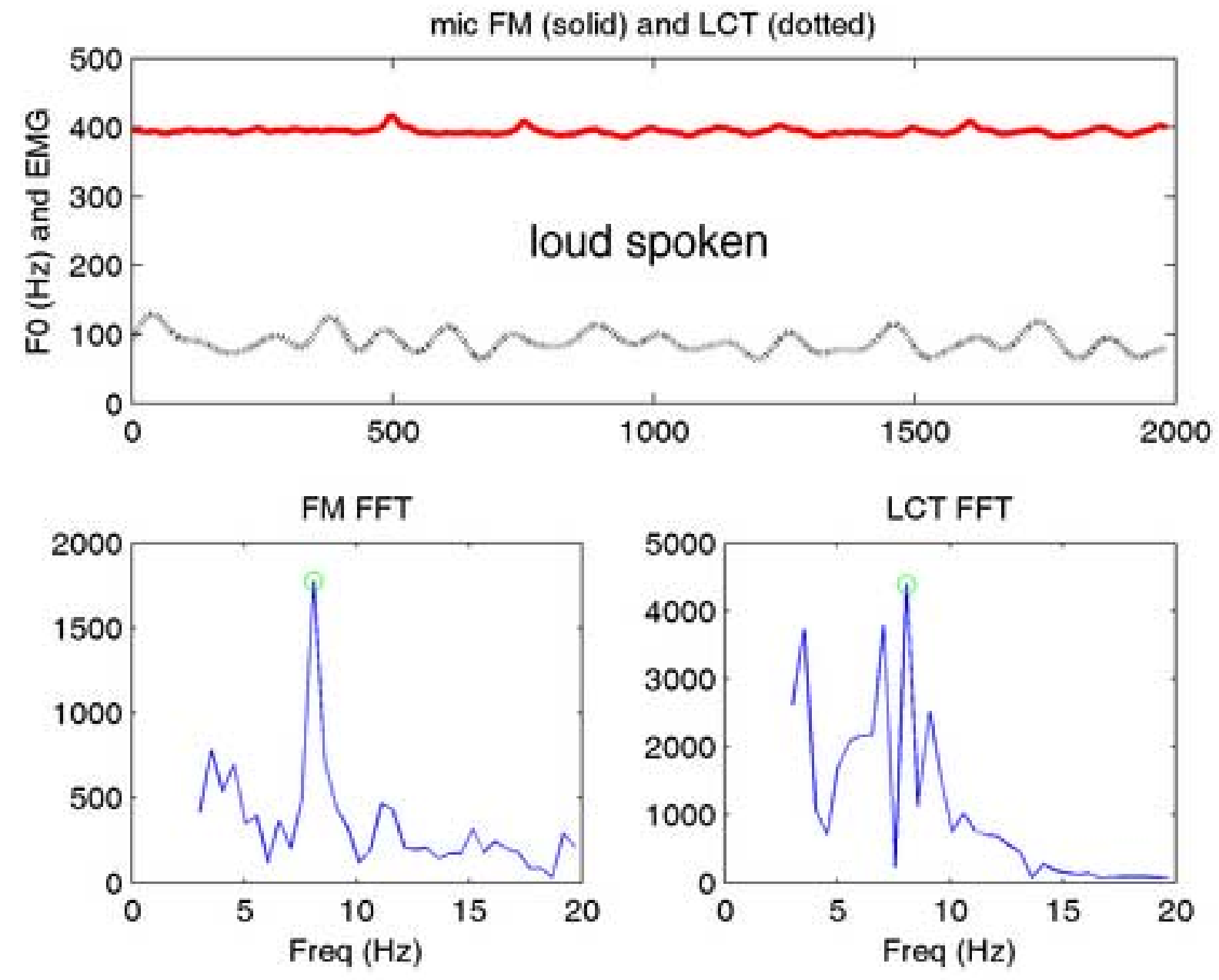